\title{
The use of convex lens as primary concentrator for multi-junction solar cells
}

\author{
Juan Paolo Lorenzo Gerardo Barrios ${ }^{1, *}$, John Raffy Cortez ${ }^{1}$, Gene Michael Herman ${ }^{1}$, Aris Larroder ${ }^{1}$, \\ Bernice Mae Yu Jeco ${ }^{2}$, Kentaroh Watanabe ${ }^{2}$, and Yoshitaka Okada ${ }^{2}$ \\ ${ }^{1}$ Philippine Science High School Western Visayas Campus, Department of Science and Technology, Bito-on, Jaro, \\ Iloilo City 5000, Philippines \\ ${ }^{2}$ Research Center for Advanced Science and Technology, The University of Tokyo, 4-6-1 Komaba, Meguro-ku, Tokyo 153-8904, \\ Japan
}

Received: 7 February 2018 / Accepted: 27 August 2018

\begin{abstract}
A concentrator lens system was designed for a multi-junction solar cell, CDO-100-C3MJ, with an added feature - a convex lens was added above the Fresnel lens in order to improve the output power of the setup and reduce the need for the use of solar trackers. The convex lens setup was tested with the Fresnel lens setup over a 3-day photoperiod by measuring the voltage, current, irradiance, and temperature at every hour. The results showed that the convex lens setup produced $1.94 \%$ more power, but only at around midday. The increase in power is due to the convex lens that focuses a greater amount of irradiance on the solar cell over the course of the day.
\end{abstract}

Keywords: multi-junction solar cell / two-lens system / concentrator / convex lens / overall performance

\section{Introduction}

Solar cells can be composed of a single pair of semiconductor materials, called single junction solar cells (SJSCs), or multiple layers of different semiconductor materials, called multi-junction solar cells (MJSCs). These MJSCs can absorb a wider spectrum of wavelengths due to the different semiconductor materials present in the cell, making it more efficient. The highest recorded efficiency (defined as the ratio of the electrical output power of the cell to the solar radiant flux) for SJSCs is only $28 \%$, while MJSCs with concentrator lenses have reached an efficiency of $46 \%$ with the latest design from Fraunhofer Institute for Solar Energy Systems [1].

Concentrators are able to reduce materials cost while at the same time increase efficiency of the solar cell by concentrating a large surface area of sunlight and its resulting heat onto a smaller solar cell, increasing its open circuit voltage by limiting entropy production during the absorption and emission of radiation [2]. The most commonly used lens concentrator is the Fresnel lens, which has been used since the 1950's when plastics were used for lenses [3]. Plastics were determined to be effective due to their thermal stability and transmissivity, which matches the solar spectrum and their index of refraction which is similar to that of glass.

\footnotetext{
* e-mail: jplgbarrios@gmail.com
}

Of the several factors affecting solar cell output power, the angle relative to the sun is one of the most important considerations in terms of collecting sunlight. The desired angle is usually achieved by mounting the solar cells on ground or roof-based mounting supports [4]. This would cause the solar cell to be fixed at a certain angle, resulting in inconsistent light incidence due to the movement of the sun [5]. This also means that the angle of the solar cell relative to the sun is dependent on its global position and the season [6]. This poses a problem since concentrator MJSCs can only absorb direct sunlight, whereas non-concentrator solar cells can absorb both direct sunlight as well as diffused sunlight [7]. This problem is addressed by aligning the MJSCs with the sun using solar trackers. These are moving mounts that adjust the angle of the solar cell to match the movement of the sun. However, using such mounts come at the cost of having additional maintenance and energy expenses [8]. In order to minimize the use of solar trackers, the optimum angle for a solar cell has been studied and was determined to be based on the solar cell's global position and the time of the year [9].

This study attempts to enable the concentrator MJSC to capture the maximum amount of sunlight without the use of solar trackers based on the output power of the cell. This requires a mechanism that would be able to gather light in multiple directions and focus them on one point. Previous researches including that of Lorenzo and Luque [10], Ning et al. [11], Akhmedov et al. [12], Donovan et al. [13], Fu et al. in 2011 [14], Xie et al. [15], and Vincenzi et al. [16] have used 

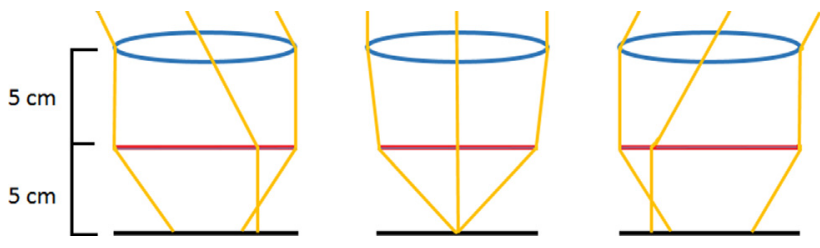

Fig. 1. The predicted ray diagram for the two-lens system.

various ways of capturing light from multiple angles, including Fresnel lens concentrators, parabolic mirrors, Cassegrain concentrators, and flat mirror concentrators. However, these methods have lower acceptance angles and higher manufacturing costs compared to two-lens systems, which have the added benefit of increasing solar concentration on the solar cell.

Two-lens systems have already been implemented in 1980's. One example is that of Ning et al. [11] which used a Fresnel lens, which is a flat lens made up of a number of concentric rings and has the benefit of being lighter and less light absorbent compared to conventional lenses while retaining their focusing ability. This lens was coupled with non-imaging concentrators (lenses that are designed only for radiative transfer as opposed to image quality) as secondaries tested with silicon solar cells. Their study found that the lens systems offered higher concentration, increased acceptance angle, and a more uniform flux distribution. However, while they may be cheaper than convex lenses due to the decreased amount of glass required per lens, it is possible for Fresnel lenses to collect more debris due to the grooves present on the surface, especially in dusty climates such as the Philippines [17]. These cause the Fresnel lenses to become less transparent, thus reducing efficiency. In addition, the use of two-lens systems with MJSCs remains largely unexplored.

This study investigated the use of convex lenses as primary concentrators, in addition to the existing Fresnel lens already used as concentrators in MJSCs to form a twolens system, as conceptualized in Figure 1. The three diagrams demonstrate the predicted behaviour of the light rays in the morning, midday, and afternoon phases of the experiment wherein the blue and red elements represent the convex and Fresnel lenses, while the black line represents the solar cell (not drawn to scale). The lens system was designed so that the primary concentrator (in this case a convex lens) would be able to refract sunlight from non-perpendicular angles to the secondary concentrator (in this case a Fresnel lens), which would then focus the sunlight onto the solar cell.

In this paper, the Methods will be presented first, followed by the limitations, results, discussion, dead end, and finally the conclusion. We will refer to the setup with the convex lens as "convex lens setup", and the setup with just the Fresnel lens as "Fresnel lens setup".

\section{Methods}

This study underwent five stages: the Fresnel lens setup assembly, convex lens setup assembly, testing of both setups, theoretical modelling, and data analysis. In the Fresnel lens setup assembly stage, a Fresnel lens was mounted above the solar cell to function as the base solar kit.

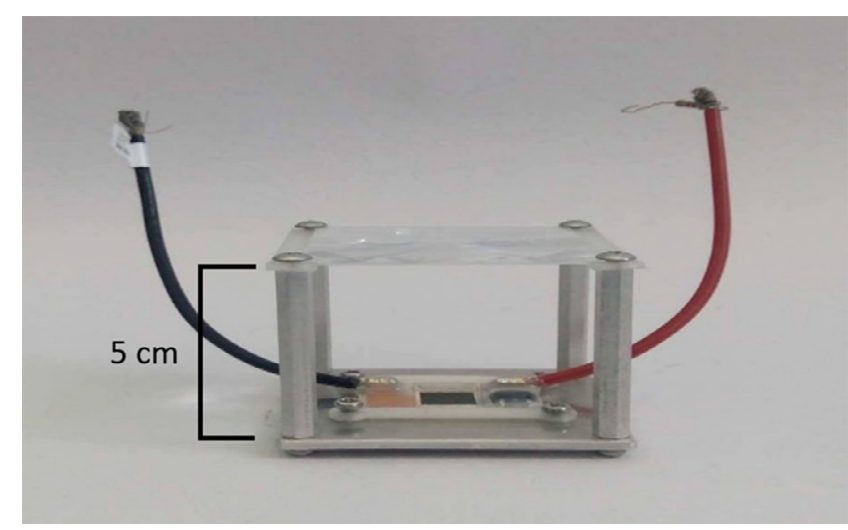

Fig. 2. The Fresnel lens setup which uses a Fresnel lens concentrator.

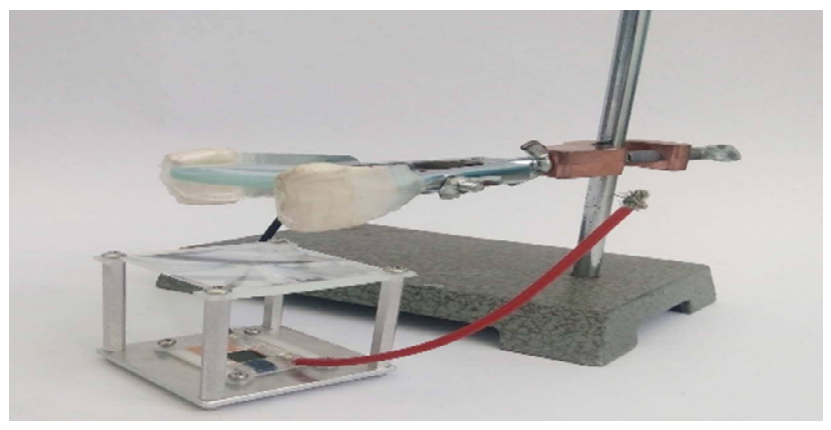

Fig. 3. The two-lens system with convex lens as primary concentrator located $5 \mathrm{~cm}$ above the Fresnel lens secondary concentrator.

\subsection{Fresnel lens setup}

The Fresnel lens setup is comprised of a Fresnel lens mounted $5 \mathrm{~cm}$ above the solar cell, as shown in Figure 2. The solar cell is placed $5 \mathrm{~cm}$ under a Fresnel lens measuring $5 \mathrm{~cm} \times 5 \mathrm{~cm}$ with a measured optical efficiency of $96.2 \%$ as measured using an irradiance meter and sourcemeter. Additionally, the lens has an estimated acceptance angle of 84.7 to $101.6^{\circ}$ relative to the lens surface as measured with a laser pointer and a protractor.

For the convex setup assembly stage, the lens kit was assembled using an iron rod and an iron clamp, such that the convex lens can be easily removed and reattached to the base solar kit.

\subsection{Convex lens setup assembly}

A convex lens with a diameter of $5 \mathrm{~cm}$, a focal length of $10 \mathrm{~cm}$, and an estimated acceptance angle of 83.7 to $97.5^{\circ}$ relative to the lens surface was selectively. It was mounted at a fixed height of $5 \mathrm{~cm}$ above the Fresnel lens using the iron stand and iron clamp, as shown in Figure 3.

The specific measurements for the lens installation were calculated using the Lens-Maker's equation (1), where the dimensions were selectively aimed at having the focal point of the two-lens system placed exactly on the solar cell's surface. $f_{1}$ and $f_{2}$ represent the focal lengths of the convex and Fresnel lenses, respectively, $d$ represents the distance 
between the two lenses, and $f$ represents the composite focal length of the entire lens system.

$$
\frac{1}{f}=\frac{1}{f_{1}}+\frac{1}{f_{2}}-\frac{d}{f_{1} f_{2}} .
$$

Afterwards, both setups were tested over a 3-day photoperiod, which refers to the time of day where there is sunlight.

\subsection{Testing of both setups}

The solar kit, with and without the convex lens attachment, was exposed to sunlight to test its output power by measuring its voltage, current, and temperature using a multimeter. This was done at every hour, from sunrise at $6: 21$ to sunset at 17:21, to measure the output power at extreme acceptance angles.

The solar kit is composed of the solar cell CDO-300C3MJ, which is comprised of Gallium Indium Phosphide, Gallium Indium Arsenide, and Germanium with an efficiency of $25.5 \%$ as measured using the Light Bias Method. This is a way of measuring the efficiency of the individual solar cell junctions by exposing the cell to lights of different wavelengths and measuring its corresponding current output. This method yields the external quantum efficiency, or the ratio of the number of electrons produced by the cell divided by the amount of photons incident on it [18].

The irradiance meter used was a Tenmars TM-206 Solar Power Meter capable of measuring irradiance up to $2000 \mathrm{~W} / \mathrm{m}^{2}$ with a resolution of $0.1 \mathrm{~W} / \mathrm{m}^{2}$. It has an accuracy of within $\pm 10 \mathrm{~W} / \mathrm{m}^{2}$ or $\pm 5 \%$, whichever of the two values is greater when the device is used, and a temperature included error of $\pm 0.38 \mathrm{~W} / \mathrm{m}^{2} /{ }^{\circ} \mathrm{C}$ deviation from $25^{\circ} \mathrm{C}$. It has a sampling time of 0.25 seconds and is cosine corrected in terms of angular accuracy.

The multimeter used was an Extech EX470A 12 Function True RMS Multimeter + IR Thermometer. It has a DC voltage measurement range from $0.1 \mathrm{mV}$ to $600 \mathrm{~V}$, DC current measurement range from $0.1 \mu \mathrm{A}$ to $10 \mathrm{~A}$, resistance measurement range from $0.1 \Omega$ to $40 \mathrm{M} \Omega$, and a temperature measurement range from -4 to $1382^{\circ} \mathrm{F}(-20$ to $750^{\circ} \mathrm{C}$ ). CAT III Test Leads were used for this experiment.

Finally, a $1 \mathrm{k} \Omega$-film resistor was also used as a load for a circuit with the solar cell as the power source.

The testing was held from 26th to 28th of February, 2016 from 6:21-17:21 inside the premises of the Philippine Science High School-Western Visayas Campus at the rooftop of the Student Learning and Research Center Building $\left(10^{\circ} 45^{\prime} 10.3^{\prime \prime} \mathrm{N} 122^{\circ} 35^{\prime} 15.8^{\prime \prime} \mathrm{E}\right)$.

The convex lens setup was compared with the standard concentrator photovoltaic setup, which is the same MJSC with only a Fresnel lens concentrator.

The irradiance meter indicated the amount of sunlight available during the time of testing. This provided us with a value for power incident to the solar cell, and also ensured uniform experimental conditions through the presence of uniform irradiance between the two solar kits. Meanwhile, the multimeter was used to measure the voltage, current, and temperature of both modules. The data gathering lasted for three consecutive days to minimize the variation

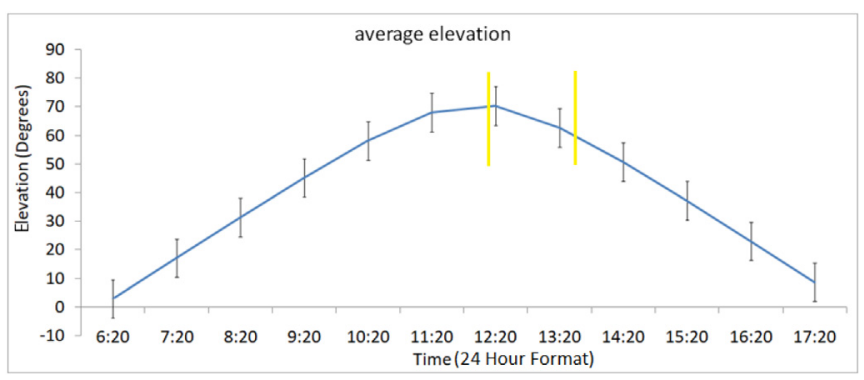

Fig. 4. The average solar elevation over the 3-day testing period. The yellow lines indicate the time period wherein the sun falls within the acceptance angle of both lenses.

in atmospheric conditions. The setup was placed on a table covered by a black doormat, so as to prevent possible interference from the light reflected from the white tabletop. The tilt of the table was also measured, which was determined to be flat and level using Multi-measure Tool Kit application which was calibrated upon usage.

In order to test the accuracy of the data gathered, theoretical modelling was done. The amount of sunlight received by the solar cell was calculated using values from the University of Oregon Solar Calculator [19] corresponding to the position of the sun during the testing period, as well as the amount of incident solar radiation taken from the Photovoltaic Geographical Information System of the European Commission Joint Research Centre [20] and the equation for lens system magnification taken from Jones [21].

\subsection{Theoretical modelling}

Values for solar elevation at every hour during the testing period were retrieved from the University of Oregon Solar Calculator, and are illustrated in Figure 4. Since the convex lens has an acceptance angle of $83.7^{\circ}-97.5^{\circ}$ relative to the lens surface and the Fresnel lens has an acceptance angle of $80.4^{\circ}-101.6^{\circ}$ relative to the lens surface, the lenses can only function as concentrators when the Solar Elevation falls within the acceptance angle. The hourly solar elevations were compared with this angle, yielding a maximum estimated time frame of sometime between 12:20 and 13:20.

Next, the latitude and longitude of the testing site were inserted into the Photovoltaic Geographical Information System of the European Commission Joint Research Centre, which yielded the average irradiance values at every hour during the month of February at the specified location. These irradiance values were then multiplied by the concentration that can be provided by the lenses in order to calculate the amount of sunlight incident on the solar cell. The individual concentrations of the lenses are simply their individual surface area measurements $(25 \times$ for the Fresnel lens and $19.63 \times$ for the convex lens), whereas the concentration for the convex lens setup can be calculated by multiplying the individual magnification of the two-lenses, represented as $M_{1}$ and $M_{2}(2)$.

$$
\text { Magnification }_{\text {total }}=M_{1} \times M_{2} \text {. }
$$


Table 1. Power incident on the solar cells measured in Watt.

\begin{tabular}{lll}
\hline Time & Fresnel & Convex \\
\hline $11: 20$ & 1.70 & 33.42 \\
$12: 20$ & 1.80 & 35.42 \\
\hline
\end{tabular}

The concentration for the convex and Fresnel lens setups was determined to be $490.87 \times$ and $25 \times$, respectively.

Multiplying the concentration by the incident irradiance yields the theoretical sunlight incident on the solar cell in each of the setups, as shown in Table 1.

\subsection{Data analysis}

The Wilcoxon Signed Ranks Test was used to compare the daily output power of both the convex and Fresnel setups. It is a non-parametric test used to compare matched samples to determine if a significant difference exists for their population mean ranks [22]. This non-parametric test was used since the data is not entirely random due to the assigned time for gathering the values, and thus cannot be assumed to be normally distributed.

Average values for voltage, current, output power, irradiance, and temperature were obtained by averaging the values at each hour for the 3-day testing period.

The output power of the solar cell was calculated using a multimeter and an irradiance meter. This can be determined from the power output, which can be derived from the voltage and current measured by the multimeter.

\subsection{Limitations}

This study is limited to measuring the output power rather than external quantum efficiency due to the lack of a sourcemeter. It is also limited to milliwatt measurements due to the small size of the setups. Measurements were taken every hour to determine the effect of the convex lens at different hours of the day. In addition, the study is also limited by our inability to control external factors such as weather conditions and cloud cover. This is compensated, however, by the 3 -day testing period and by appropriate scheduling of the testing dates with respect to weather forecasts.

\section{Results}

The study aimed to design a solar cell setup with a convex lens as a primary concentrator, coupled with a Fresnel lens as a secondary concentrator and to test the output power of the Fresnel and Convex lens setups by exposing them to sunlight at every hour during the photoperiod to measure output power across varying angles of sunlight as well as irradiance.

\subsection{Voltage, current, irradiance, and temperature}

The irradiance values in Figure 5 are consistent with what is expected in terms of the photoperiod, wherein midday is

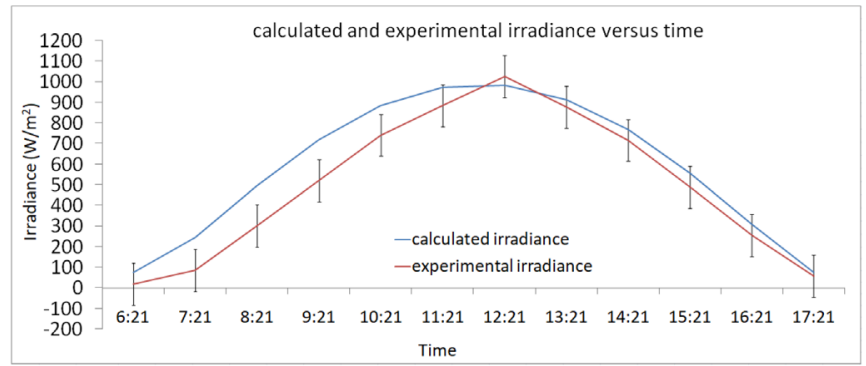

Fig. 5. Irradiance versus time graph over the 3-day data gathering period. The irradiance increased until mid-day, 12:21, where was at its maximum and decreased until 17:21.

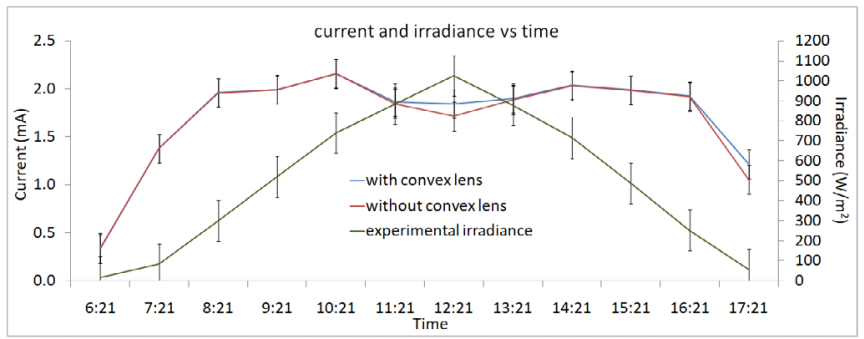

Fig. 6. Average voltage versus time graph from 6:21 to 17:21

the hottest part of the day. Irradiance values increased until midday and then decreased until the end of the photoperiod. In comparison to the theoretical values, the experimental measurements are lower from 6:21 to 9:21 and 15:21 to $17: 21$. On the other hand, the experimental measurements are higher than the theoretical values from 9:21 to 15:21. The lower experimental values are caused by cloud cover, as the theoretical values assume perfectly clear skies. As for the increase in experimental values from 9:21 to $15: 21$, this could be due to the difference in the amount of sunlight permeating the atmosphere in comparison to the constant used in equation (2).

The voltages of the two setups rapidly increased from 6:21 to 8:21 as seen in Figure 6 due to an increase in the amount of sunlight being received by the solar cell. From 8:21 to $11: 21$, the voltage gradually decreased but after 12:21, the voltage again increased until around 16:21 and then rapidly decreased from 16:21 to 17:21 due to a decrease in sunlight received by the cell. The voltages for the two setups are similar throughout the photoperiod except around 12:21 where the convex lens setup produced a higher voltage. It appears that the solar cell has a maximum voltage of around $2.5 \mathrm{~V}$, as this is where the voltage stops increasing. A slight decrease in voltage can be observed during the time when the sun falls within the lens system's acceptance angle, which could be caused by lens reflection.

Similar to the voltage versus time graph, Figure 7 shows that the two setups also performed similarly in terms of current except around 12:21 and around 16:21-17:21, wherein the convex lens setup produced a larger current. The reason behind this behaviour is the same as that for 


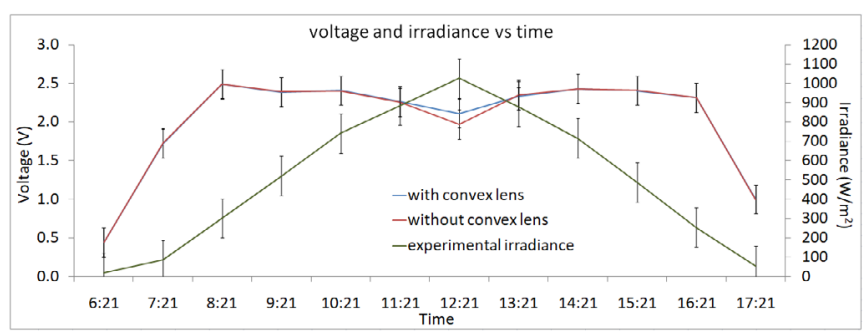

Fig. 7. Current versus time graph from 6:21 to 17:21 over the 3 -day data gathering period. The values obtained from the two setups does not differ much except at around 11:21-13:21 and $16: 21-17: 21$.

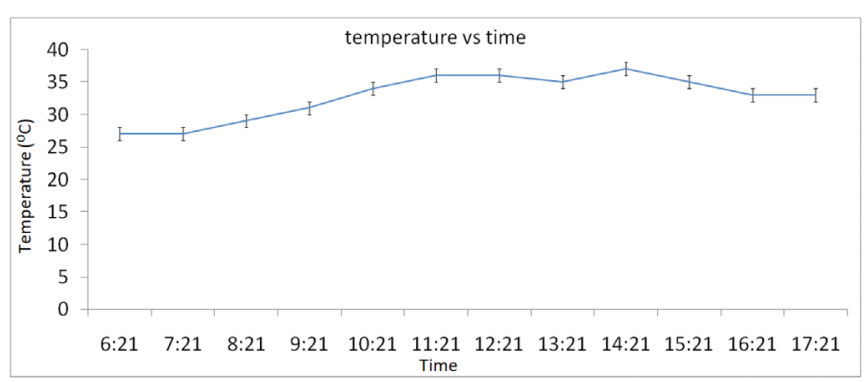

Fig. 8. Temperature versus time graph over the 3-day data gathering period. The maximum measured temperature was at around 14:21. The trend however is not uniform which is caused by the weather.

voltage, wherein the increase and decrease in sunlight as the day progresses affect the voltage and current output. Note that a slight increase in current can also be observed from 15:21 to 17:21 for the convex lens setup, the reason for which could be due to a slight difference in cloud cover.

The temperature changes in Figure 8 are affected by the irradiance, wherein higher irradiance induces higher cell temperatures due to the greater amount of sunlight incident on the cell.

The two solar cell setups performed comparably throughout the photoperiod, except at around 12:21, where the convex lens produced more power. The power itself rose from 6:21 to 10:21, decreased at 12:21, and then increased again until 14:21 then it began to decrease as the photoperiod ended.

In terms of irradiance, the convex lens setup performed better at irradiances exceeding $800 \mathrm{~W} / \mathrm{m}^{2}$, as it was also the time that the alignment of the sun was within the acceptance angle of the lens. However, the output power of both solar cells throughout other irradiance values appeared to be comparable. In terms of total power output throughout the day, the convex lens setup produced $1.94 \%$ more power compared to the Fresnel lens setup.

\subsection{Data analysis}

The Wilcoxon Signed Ranks Test was used to determine the significance of the difference in power between the two setups. The test requires a $p$-value (asymp. sig. 2-tailed) or calculated probability that is less than 0.05 for the result to
Table 2. $p$-Value for the difference in power outputs between the convex and Fresnel lens setups at midday and the whole day.

\begin{tabular}{lll}
\hline Diff. in power & $Z$ & Asymp. sig. (2-tailed) \\
\hline Midday & -2.032 & 0.042 \\
Wholeday & -2.051 & 0.040 \\
\hline
\end{tabular}

be significant. Table 2 shows the $p$-values for the difference in power between the convex and Fresnel lens setups at midday and for the whole photoperiod, respectively. The $Z$ value indicates the $z$-score, which is the number of standard deviations that the power difference is from the mean power.

Table 2 indicates that the $p$-value is less than 0.05 for both the hourly and daily power output. This means that the difference in power output is significant. The $Z$ values in both mid and whole day are similar, at around -2 . This indicates that the difference in power outputs is around 2 standard deviations below the mean power output.

\section{Discussion}

The results showed that the convex lens setup has a higher power output at around 11:21-13:21 compared to the Fresnel lens setup. This could be attributed to the acceptance angle of both setups, which only directs sunlight to the solar cell at this time of the photoperiod. This could also be the primary reason why the convex lens setup had a greater daily total power output compared to the Fresnel set-up: the increase in irradiance due to the refraction and concentration from the convex lens. Since the compound focus of the lens system is on the solar cell, the image produced will overlap which in this case is the light from the sun making it more concentrated. Although it was estimated that the acceptance angle of the convex lens setup was smaller than that of the Fresnel lens, it appears that the increase in concentration compensates for that since the power output for the convex lens is still higher than that of the Fresnel lens setup. It is observed however that there is a decrease in current, voltage, and power output coinciding with the time period when the sun fell within the acceptance angles of the lenses. This seems to suggest that the lens reflects some of the sunlight, causing a decrease in power input for the solar cell.

Discrepancies between experimental and theoretical irradiance values can be seen in Figure 5. An average error of $87 \%$ was recorded for the convex lens setup from 12:20 to 13:20 and $87.5 \%$ on the Fresnel lens setup for the same time period. These deviations were primarily caused by the difference of the experimental and theoretical values for the irradiance since power is dependent on irradiance. This difference can be attributed to the presence of clouds, which are not taken into account in the theoretical calculations. A cloud cover protocol exists and was used in this experiment. Unfortunately, this method is mostly qualitative in nature and requires visual estimates which will most likely result in errors. 
The increase in the power output with respect to the irradiance is also similar, wherein higher irradiance values also induced higher power outputs in both cells, albeit slightly higher in the convex lens setup. This indicates that the amount of irradiance absorbed by the solar cell itself increased.

Maximum values for voltage and current can be observed in Figures 6 and 7, wherein the values for both do not increase despite the increasing amount of sunlight received by the solar cell. These values are approximately $2.5 \mathrm{~V}$ and $2.2 \mathrm{~mA}$, respectively.

As observed in Figure 7, a slight current increase can be observed from 15:21 to 17:21. Upon comparison between the theoretical and experimental irradiance values, this could be attributed to a difference in cloud cover. This is one of the limitations of our study: the unavailability of dark rooms and solar simulators that are used in most solar cell studies.

The Wilcoxon Signed Ranks Test showed that there is a significant difference in power outputs both for the hourly comparison between the two setups as well as the total power output at the end of the photoperiod. Although the difference was small for the time before and beyond 10:21$14: 21$, the large difference at the peak of the sunlight compensates for the small difference. The size of the solar cells and the overall setups must be considered. Since the lenses and solar cells used are small, then it can be expected that the differences in output power will also be small. Further tests with larger setups must be performed to better test and determine if the difference in power outputs is indeed significant.

Considering that the Philippines is a tropical country, and that the solar irradiance is fairly consistent throughout the year, the output power measured should be consistent regardless of what the time of the year when it is tested.

\subsection{Error analysis}

Several factors have been identified that could have affected the results of the study. The first of which is parallax error which could have occurred during the mounting/unmounting of the convex lens. This is a systematic error, and would be the same throughout the entirety of the test since the same point of view (top view) was used when aligning the lenses, and markings on the table and iron stand were used as references when mounting and demounting the convex lens. Another factor is external sources of refraction. While the work area was secured by the researchers to limit this factor as much as possible, this random error cannot be escaped completely. Third is possible defects in the lenses or the solar cell itself. While the lenses and solar cell were visually inspected for scratches and damage before the tests, possible internal defects cannot be ruled out. The solar cell for instance has been evaluated in terms of efficiency, but these may have changed in the time before the study was conducted. These would be systematic factors that would have been constant in the course of the study. Finally, there is the calibration of the irradiance meter and multimeter. The solar meter came with a certificate of calibration, and the researchers had no

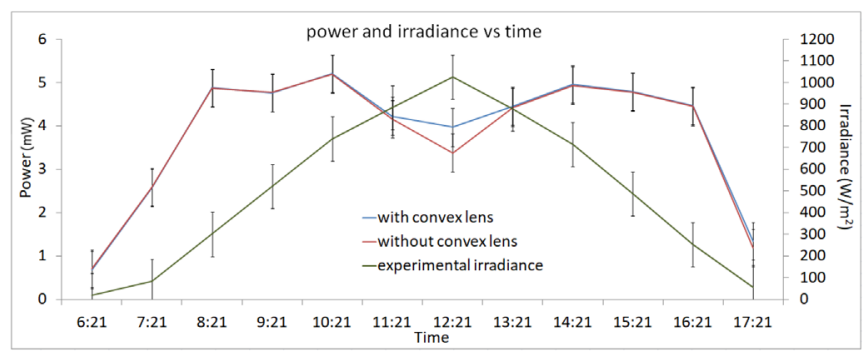

Fig. 9. Average power at every hour for the 3-day testing period. The value for power is similar to that of voltage and current, wherein the two setups performed similarly except around 11:2113:21 where the convex lens setup produced higher voltage and current. The difference in current after 16:21 that was seen in the current versus time graph is no longer evident here.

way of checking for accuracy, while the multimeter was calibrated by the school's science research assistant. Should there be defects, however, these would be systematic errors that would be consistent throughout the experiment.

\section{Dead end}

Although the study was a success, certain variables might be considered in order to improve it. The power is dependent on the irradiance and comparing the theoretical values of irradiance to the measured values, a $14.56 \%$ error was measured. This was due to the cloud cover as it affects the irradiance. Another reason might be the constant used in the calculation of theoretical irradiance, which is lower than the actual irradiance from 9:21 to 15:21. This might be caused by global warming, which is not taken into account in the theoretical irradiance calculations. Problems like this can be addressed in future studies through the use of solar simulating light sources in testing the solar cells. It was also found that the acceptance angle of the convex lens setup was smaller than that of the Fresnel lens setup. However, the results still show that the convex lens setup produces a greater output power. The measurements of the lens' acceptance angles were also found to be asymmetrical. Despite the authors' best efforts in measuring the acceptance angle using a laser pointer and a protractor, it is entirely possible that there has been an error in this process. Figures 6, 7, and 9 in Section 3 seem to show that the lens does in fact perform symmetrically however. Sadly there has not been a comprehensive analysis on the effect of the solar cell temperature recorded throughout the experiment, and as a result it is unknown how temperature affects the output power of the convex and Fresnel lens setups.

\section{Conclusion}

It was found that the convex lens setup produces a $1.94 \%$ greater amount of power compared to the Fresnel lens setup. For future study, it is recommended that the sizes of the setups be altered so as to determine whether 
the significance of the increase in power is dependent on size.

We would like to thank the following organizations for aiding us during the course of our study: The National Institute of Physics (NIP) in the University of the Philippines Diliman; San Carlos Clean Energy, Inc.; West Visayas State University; and School of Science and Engineering, Ateneo de Manila University.

\section{References}

1. High Concentration III-V Multijunction Solar Cells. National Renewable Energy Laboratory. https://www.nrel.gov/ pv/high-concentration-iii-v-multijunction-solar-cells.html (Accessed March 30, 2017)

2. L. Weinstein, J. Loomis, B. Bhatia, D. Bierman, E. Wang, G. Chen, Concentrating solar power, Chem. Rev. 115, 12797-2838 (2015)

3. R. Leutz, A. Suzuki, Nonimaging Fresnel Lenses: Design and Performance of Solar Concentrators, Springer Verlag, Heidelberg, 2001

4. United States. Massachusetts Department of Energy Resources. Massachusetts Clean Energy Center. Questions \& Answers Ground-Mounted Solar Photovoltaic Systems. June 2015. https://www.mass.gov/files/documents/2016/ 08/rn/solar-pv-guide.pdf (Accessed September 7, 2017)

5. M. Guo, Z. Haixiang, G. Shengyu, C. Tingji, X. Jing, C. Lexiang, W. Zhinong, S. Guoqiang, Optimal tilt angle and orientation of photovoltaic modules using HS algorithm in different climates of China, Appl. Sci. 7, 10 (2017)

6. H. Mousazadeh, A. Keyhani, A. Javadi, H. Mobli, K. Abrinia, A. Sharif. A review of principle and sun-tracking methods for maximizing solar systems output, Renew. Sustain. Energy Rev. 13, 1800-1818 (2009)

7. Concentrating Photovoltaics (CPV). Concentrating Photovoltaics|Solar Power. 2013. http://www.greenrhinoenergy. com/solar/technologies/pv concentration.php (Accessed September 08, 2016)

8. S. Kurtz, Opportunities and Challenges for Development of a Mature Concentrating Photovoltaic Power Industry, Technical paper no. NREL/TP-5200-43208. National Renewable Energy Laboratory, U.S. Department of Energy, Office of Energy Efficiency \& Renewable Energy, November 2012. https://www.nrel.gov/docs/fy13osti/43208.pdf (Accessed February 2, 2017)

9. Solar Angle Calculator. Solar Electricity Handbook. 2016. http://solarelectricityhandbook.com/solar-angle-calculator. html (Accessed September 24, 2016)
10. E. Lorenzo, A. Luque, Comparison of Fresnel lenses and parabolic mirrors as solar energy concentrators, Appl. Opt. 21, 1851 (1982)

11. X. Ning, R. Winston, J. O'Gallagher, Dielectric totally internally reflecting concentrators, Appl. Opt. 26, 300-305 (1987)

12. K. Akhmedov, R. Zakhidov, S. Klychev, Optical and energy characteristics of round Fresnel lenses with flat bands, Appl. Solar Energy 27, 43-46 (1991)

13. M. Donovan, B. Bourne, J. Roche, Efficiency vs. Irradiance Characterization of PV Modules Requires Angle-of-incidence and Spectral Corrections. 2010 35th IEEE Photovoltaic Specialists Conference, June 20, 2010. doi:10.1109/ pvsc.2010.5615838 (Accessed September 20, 2016)

14. L. Fu, R. Leutz, H. Annen, Evaluation and Comparison of Different Designs and Materials for Fresnel Lens-based Solar Concentrators, Nonimaging Optics: Efficient Design for Illumination and Solar Concentration VIII, 2011th ser., 81240E, September 21, 2011. doi:10.1117/12.893390 (Accessed January 5,$2017 ; 2011)$

15. W. Xie, Y. Dai, R. Wang, K. Sumathy, Concentrated solar energy applications using Fresnel lenses: a review, Renew. Sustain. Energy Rev. 6, 2588-2606 (2011)

16. D. Vincenzi, S. Baricordi, S. Calabrese, M. Musio, A. Damiano, A Cassegrain Concentrator Photovoltaic System: Comparison between Dichroic and Multijunction Photovoltaic Configurations., IECON 2014 - 40th Annual Conference of the IEEE Industrial Electronics Society, October 29, 2014. doi:10.1109/iecon.2014.7048761 (Accessed March 10, 2017)

17. C. Miguel, Air Pollution: A Public Health Concern in the Philippines, Department of Environment and Natural Resources, September 24, 2017. http://www.denr.gov.ph/ news-and-features/latest-news/3295-air-pollution-a-publichealth-concern-in-the-philippines.html (Accessed April 16 , 2018)

18. F. Liu, Y. Chang, S. Kaiwen, Z. Fangzhou, H. Xiaojing, A. Martin, Light-bias-dependent external quantum efficiency of kesterite $\mathrm{Cu}_{2} \mathrm{ZnSnS}_{4}$ solar cells, ACS Photonics 4, 1684-690 (2017)

19. Solar Position Calculator. UO SRML: Solar Position Calculator. January 8, 2016. http://solardat.uoregon.edu/ SolarPositionCalculator.html (Accessed January 15, 2017)

20. Average Daily Solar Irradiance. European Commission Joint Research Centre. http://re.jrc.ec.europa.eu/pvgis/apps4/ pvest.php?lang =en (Accessed August 13, 2018)

21. M. Jones, Lecture 31-Geometric Optics, Lecture, Purdue University. http://www.physics.purdue.edu/jones105/ phys42200_Spring2013/notes/Phys42200_Lecture31.pdf

22. F. Wilcoxon, Probability tables for individual comparisons by ranking methods, Biometrics 3, 119 (1947)

Cite this article as: Juan Paolo Lorenzo Gerardo Barrios, John Raffy Cortez, Gene Michael Herman, Aris Larroder, Bernice Mae Yu Jeco, Kentaroh Watanabe, Yoshitaka Okada, The use of convex lens as primary concentrator for multi-junction solar cells, Emergent Scientist 2, $5(2018)$ 